\title{
Cellular characteristics of primary and im mortal canine embryonic fibroblast cells
}

\author{
Seungkwon You ${ }^{1,2,6}$, Jai-Hee Moon ${ }^{1,2}$, \\ Tae-Kyung Kim ${ }^{1,2}$, Sung-Chan Kim ${ }^{3, *}$, \\ Jai-Woo Kim ${ }^{4}$, Du-Hak Yoon ${ }^{5}$, \\ Sungwook Kwak ${ }^{3}$, Ki-Chang Hong ${ }^{2}$, \\ Yun-Jaie $\mathrm{Choi}^{3}$ and Hyunggee $\mathrm{Kim}^{1,2}$ \\ ${ }^{1}$ The Laboratory of Cell Growth and \\ Function Regulation \\ ${ }^{2}$ College of Life and Environmental Sciences \\ Korea University, Seoul, Korea \\ ${ }^{3}$ School of Agricultural Biotechnology \\ Seoul National University \\ Seoul, Korea \\ ${ }^{4}$ Institute of Biotechnology, Yeungnam University \\ Gyeongsan, Korea \\ ${ }^{5}$ National Livestock Research Institute \\ Suwon, Korea \\ ${ }^{6}$ Corresponding authors: Tel, 82-2-3290-3057; \\ Fax, 82-2-3290-3507; E-mail, bioseung@korea.ac.kr \\ *Present address: Department of Biochemistry, University of \\ Texas, Southwestern Medical Center, Dallas, TX, USA
}

Accepted 16 July 2004

Abbreviations: $\mathrm{CaEF}$, canine embryonic fibroblast; PDL, population doubling level; ROS, reactive oxygen species; RT-PCR, reverse transcription-PCR

\footnotetext{
Abstract

Using normal canine embryonic fibroblasts (CaEF) that were shown to be senescent at passages $7_{\text {th }} 9_{\text {th }}$, we established two spontaneously immortalized CaEF cell lines (designated CGFR-Ca-1 and -2) from normal senescent CaEF cells, and an immortal CaEF cell line by exogenous introduction of a catalytic telomerase subunit (designated CGFR-Ca-3). Immortal CGFR$\mathrm{Ca}-1,-2$ and -3 cell lines grew faster than primary CaEF counterpart in the presence of either $0.1 \%$ or $10 \%$ FBS. Cell cycle analysis demonstrated that all three immortal CaEF cell lines contained a significantly high proportion of S-phase cells compared to primary CaEF cells. CGFR-Ca-1 and -3 cell lines showed a loss of p53 mRNA and protein expression leading to inactivation of $p 53$ regulatory function, while the
}

CGFR-Ca-2 cell line was found to have the inactive mutant $p 53$. Unlike the CGFR-Ca-3 cell line that down-regulated $\mathrm{p} 16^{\text {1NK4a }} \mathrm{mRNA}$ due to its promoter methylation but had an intact p $16^{\text {INK4a }}$ regulatory function, CGFR-Ca-1 and -2 cell lines expressed $p 16^{\text {INK4a }}$ mRNA but had a functionally inactive $p 16^{\text {INK4a }}$ regulatory pathway as judged by the lack of obvious differences in cell growth and phenotype when reconstituted with wild-type $\mathrm{p} 16^{\text {INK4a }}$. All CGFR-Ca-1, -2 and -3 cell lines were shown to be untransformed but immortal as determined by anchorage-dependent assay, while these cell lines were fully transformed when overexpressed oncogenic $H-r a s^{G 12 V}$. Taken together, similar to the nature of murine embryo fibroblasts, the present study suggests that normal primary $\mathrm{CaEF}$ cells have relatively short in vitro lifespans and should be spontaneously immortalized at high frequency.

Keywords: canine embryonic fibroblast; immortalization; $\mathrm{p} 53 ; \mathrm{p} 16^{\text {INK4a; }}$; telomerase

\section{Introduction}

Most normal diploid cells exhibit a limited replicative lifespan, termed replicative senescence (Hayflick and Moorehead, 1961; Kim et al., 2002a). With each cell division, telomeres shorten due to the inability of DNA polymerase to fully replicate the ends of linear chromosomes (Greider, 1996; Sedivy, 1998; Aviv and Harley, 2001). Accumulation of reactive oxygen species (ROS) is also associated with triggering replicative senescence (Sherr and DePinho, 2000).

Spontaneous immortalization is a rare event in human cells, but occurs much more frequently in rodent cells (Campisi, 1999; Sherr and DePinho, 2000). Common genetic alterations frequently observed in the immortalized cells are either associated with the loss of $p 53$ and $\mathrm{Rb}$ regulatory functions that are linked with cell-cycle arrest and cell death or the activation of telomerase that maintains telomere integrity or both (Bodnar et al., 1998; Sherr and DePinho, 2000; Kim et al., 2002b).

Tumor suppressor p53 inactivation via its mRNA down-expression, point mutation or deletion was found in many immortalized and tumor cell lines derived from a variety of species, with functional loss 
greater than $50 \%$ of all human tumors. In contrast to normal growth conditions in which the p53 protein does not allow cells to be growth-arrested or die in response to DNA damage, the functional activity of p53 as a transcriptional activator increases via posttranslational modifications (phosphorylation and acetylation). This increase in turn induces the expression of a number of genes that are involved in cell cycle arrest and apoptosis, including p21 WAF1 $($ El-Diery et al., 1993), Bax (Miyashita et al., 1995), PERP (Attardi et al., 2000) and Noxa (Oda et al., 2000), as well as MDM-2, p53's negative-feedback regulator (Barak et al., 1993; Wu et al., 1993; Wood and Vousden, 2001).

The $\mathrm{Rb}$ tumor suppressor pathway is mainly regulated through phosphorylation and dephosphorylation of the $\mathrm{Rb}$ protein by a complex of D-type cyclins and cyclin-dependent kinases $4 / 6$ (CDK4/6) in the G1 cell cycle stage and by $\mathrm{p} 16^{\text {INK4a }}$, an inhibitor of CDK4/6 (Serrano et al., 1993; Dyson, 1998). Many immortal and tumor cells have been shown to have a functional loss of $\mathrm{Rb}$ regulatory pathways due to either $\mathrm{Rb}$ mutation or downexpression of $\mathrm{p} 16^{\text {INK4a }}$ mRNA by its promoter methylation and/or its gene deletion.

Numerous studies have been shown that functional loss of both the $\mathrm{p} 53$ and $\mathrm{p} 16^{\mathrm{INK} 4 \mathrm{a}} / \mathrm{Rb}$ pathways as well as the upregulation of telomerase or the maintenance of telomere integrity are necessary for cellular immortalization. Here, we report (1) the cellular nature of primary canine embryonic fibroblast (CaEF), (2) the spontaneous immortalization of $\mathrm{CaEF}$ cell lines (designated CGFR-Ca-1 and -2) and (3) characterization of the cellular physiological alterations occurring in CGFR-Ca1 and -2 in comparison with those of primary $\mathrm{CaEF}$ and hTERT-mediated immortal CaEF (CGFR-Ca-3).

\section{Materials and Methods}

\section{Cells and culture conditions}

Primary $\mathrm{CaEF}$ and all three immortal $\mathrm{CaEF}$ cell lines as well as human embryonic fibroblast (HEF) and human 293T cells were grown in DMEM/high glucose enriched with $10 \%$ FBS, $1 \%$ penicillin-streptomycin, and $2 \mathrm{mM}$ L-glutamine. All cell culture reagents were purchased from Invitrogen (Carlsbad, CA). The immortalized CGFR-Ca-1, -2 and -3 cell lines were obtained spontaneously via several passages of cells from five different canine embryonic muscle tissues (CGFR-Ca-1 and -2 from two different canine embryos) and from hTERT-derived (CGFR-Ca-3) cell lines.

\section{Cell growth}

To determine cellular lifespan, primary passage 4 th CaEF cells were plated at a density of $3 \times 10^{5}$ cells $/ 10$ $\mathrm{cm}$ dish and passaged every 3 days following the standard 3T3 protocol, and the population was determined to have doubled each successive day. Primary CaEF cells were subjected to senescenceassociated $\beta$-galactosidase (SA- $\beta$-gal) assay (Dimri et al., 1995) and standard Giemsa staining to determine replicative senescence rates and to visualize cell colonies, respectively. Cell growth curve was also determined by plating cells at a density of $10^{4}$ cells $/ 6$ well dish using conditions as described above and staining cells with $0.01 \%$ crystal violet solution everyday for 6 days. Crystal violet from stained cells was extracted using $10 \%$ acetic acid and subjected to spectrophotomeric analysis $(600 \mathrm{~nm})$ to determine relative cell growth rates. Cell cycle analysis was carried out using FACS of propidium iodide-stained cells and a Cell Quest software program (Becton Dickinson) as described (Felsher and Bishop, 1999). To determine density-dependent growth rates, primary CaEF and three immortal CGFR-Ca-1, -2 and -3 cell lines were plated at a density of 300 cells per each of 6-well dish. Fourteen days after plating, all cells were subjected to staining with $0.01 \%$ crystal violet solution.

\section{Retroviral-mediated infection}

To prepare retroviruses containing $p B a b e-p u r o m y c i n$, pBabe-hygromycin, pBabe-p53WT-hygromycin, pBabep16 ${ }^{\text {INK4a }}$-puromycin and pBabe-H-Ras ${ }^{\text {V12 }}$-puromycin vectors, these vectors were transfected into the PT67 packaging cell line (Clontech) using Lipofectamine 2000 (Invitrogen) and selected with appropriate antibiotics ( $3 \mu \mathrm{g} / \mathrm{ml}$ puromycin and $200 \mu \mathrm{g} / \mathrm{ml}$ hygromycin). Supernatants of the stably transfected PT67 cells ( $>90 \%$ confluent) were filtered through a 0.45 $\mu \mathrm{m}$ filter to remove cellular debris, and then used to infect primary CaEF and immortal CGFR-Ca-1, -2 and -3 cell lines. These cells were subjected to infections 2-3 times with each fresh PT67 supernatant at $10 \mathrm{~h}$ intervals and then selected with appropriate antibiotics (1 $\mu \mathrm{g} / \mathrm{ml}$ puromycin and $100 \mu \mathrm{g} / \mathrm{ml}$ hygromycin).

\section{Adriamycin and 5-aza-2'-deoxycytidine treatment}

Primary CaEF and immortal CGFR-Ca-1, -2 and -3 cell lines were treated with the DNA-damaging agent adriamycin $(0,20,200$ and 2,000 nM) for 3 days, and then subjected to cell viability assay. For DNA methylation assay, the primary $\mathrm{CaEF}$ and three immortal CaEF cell lines were treated with DNA-methylation inhibitor 5-aza-2'-deoxycytidine $(10 \mu \mathrm{M})$ for 3 days, and then the total RNA from these cells was isolated using TRIzol (Invitrogen).

\section{Semi-quantitative reverse transcription- PCR (RT-PCR)}

For semi-quantitative RT-PCR, $3 \mu \mathrm{g}$ of DNase I-trea- 
ted RNA was converted to cDNA using Superscript II reverse transcriptase (Invitrogen) following the manufacturer's instructions. A portion $(1 \mu \mathrm{l})$ of the reverse transcription reaction was used to amplify p53, p16 ${ }^{\text {INK4a }}$ and GAPDH cDNA fragments with canine-specific primers [canine p53 (AF060514), forward primer: 5'-GCTTTGAGGTACGCGTTTG-3', reverse primer: 5'-CACGGATCTGAAGGGTGAA-3'; canine p16 ${ }^{\text {INK4a }}($ AF234176), forward primer: 5'-CTTCCTGGAC ACGC T-3', reverse primer: 5'-AGGTACCGTGCGACA-3'; canine GAPDH (AB038240), forward primer: 5'-GATTGTCAGCAATGCCTCCT-3', reverse primer: 5'-AAGCAGGGATGATGTTCTGG-3']. PCR amplifications were verified to be in the linear range.

\section{Western blot analysis}

Total cell extract was isolated from both the exponentially-growing primary $\mathrm{CaEF}$ and the three immortal CaEF cell lines using a RIPA buffer containing $1 x$ Protease inhibitor cocktail (Roche). Cell extracts (20 $\mu \mathrm{g})$ were resolved on a $4-12 \%$ gradient precasted SDS-PAGE (Invitrogen) and transferred onto PVDF membranes (Invitrogen). Immunoblotting of the membranes was performed using 1:500 dilution of anti-p53 antibody (sc-99;PAB260, Santa Cruz Biotechnology or Ab1;Pab260, NeoMarkers) and $1: 2,500$ dilution of anti- $\alpha$-tubulin antibody (Sigma), followed by treatment with HRP-conjugated anti-mouse IgG antibody (Pierce). Both canine p53 and $\alpha$-tubulin were detected by the SuperSignal West Pico kit (Pierce).

\section{Oncogenic transformation}

To determine the transforming properties of primary CaEF and immortal CGFR-Ca-1, -2 and -3 cell lines that were infected with either pBabe-puromycin or pBabe-H-Ras ${ }^{\mathrm{V} 12}$-puromycin vector, all infected cells $\left(10^{4}\right.$ cells) were plated in 6-well soft-agar dishes ( $1.6 \%$ and $0.7 \%$ bottom and top agar, respectively) and incubated at $37^{\circ} \mathrm{C}$ for 14 days.

\section{Results}

\section{Cellular lifespan and spontaneous immortal conversion of $\mathrm{CaEF}$ cells}

We determined the in vitro lifespan of primary $\mathrm{CaEF}$ cells using standard 3T3 assay. The growth rates of the primary CaEF cells thawed at passage 4 averaged 0.36 population doubling per day (PDL) at passages 5,6 and 7 (Filgure 1A). As shown in Figures $1 A$ and $B$, however, primary $C a E F$ cells were found to reach the senescent stage at passage 8 , which is indicative of the relatively short lifespan, similar to murine and bovine embryonic fibroblast (MEF) cells (Kim et al., 2002a; You et al., 2004). Reminiscent to MEF cells, a number of primary CaEF cells frequently developed small colonies of cells from the senescent cell population at passages 8 to 10 in which a majority of primary $\mathrm{CaEF}$ cells showed enlarged, flattened, $\beta$-galactosidase-positive, and highly vacuolated morphologies, phenotypical of senescent fibroblast (Figure 1B). Immortalized $\mathrm{CaEF}$ cells were eventually established from most of the cellular colonies in the senescent cell population, indicating spontaneous cellular conversion (Filgure 1C).

As shown in Figure 1D, the immortal CGFR-Ca-1, -2 (both cell lines spontaneously established from primary CaEF cells) and -3 (derived from primary CaEF cells transduced with human TERT) cell lines appeared to be relatively smaller, brighter and more uniform compared to presenescent passage $3 \mathrm{CaEF}$ cells, which displayed a spindle-shaped morphology, phenotypical of fibroblasts.

\section{Cell growth rates}

The growth curve for the primary $\mathrm{CaEF}$ and three immortal CaEF cell lines is shown in Figure 2A. All three immortal CaEF cell lines (CGFR-Ca-1, -2 and -3) grew significantly faster than the primary $C a E F$ cells. The cell growth in CGFR-Ca-2 decreased slightly at day 4 compared to that at day 2 , due to cellular properties of CGFR-Ca-2, when it reached confluence. The CGFR-Ca-2 cell line was shown to be easily detached from the substrate of the culture dish (Figure 2B), and the detached CGFR-Ca-2 cell line was likely to be dead as determined by trypan blue exclusion (data not shown). Similarly, primary CaEF cells also appeared not to become confluent, but to be detached from the culture dish (Figure 2B). In contrast to the primary $\mathrm{CaEF}$ and cell line CGFRCa-2, CGFR-Ca-1, and CGFR-Ca-3 were found to become fully confluent. Cell cycle analysis demonstrated that all three immortal CaEF cell lines contained a significantly high proportion of S-phase cells compared to primary CaEF cells (Figure 2C). Under the $0.1 \%$ FBS-DMEM culture condition, all three immortal CaEF cell lines were shown to grow faster than their primary $\mathrm{CaEF}$ counterparts. It is noteworthy that the growth rate of the three immortal CaEF cell lines in $0.1 \%$ FBS-DMEM were similar to primary CaEF cells growing in the 10\% FBS-DMEM (Figure 2D). In addition, when cells were allowed to grow in low cell density, all three immortal CaEF cell lines were found to grow faster and generate larger colonies compared to primary $\mathrm{CaEF}$ cells (Figure 2E). Taken together, immortal CGFR-Ca-1, -2 and -3 cell lines seem to obtain a fast-growing property during spontaneous or hTERT-mediated immortal conversions. 


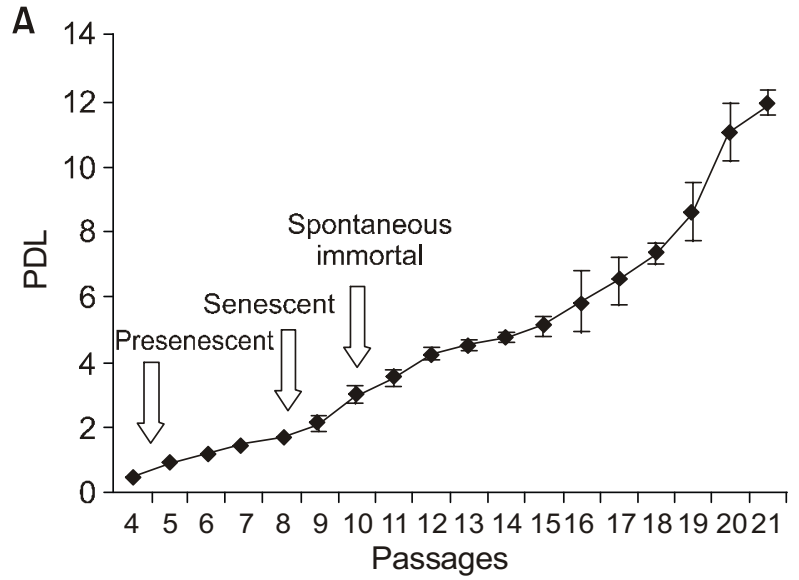

B

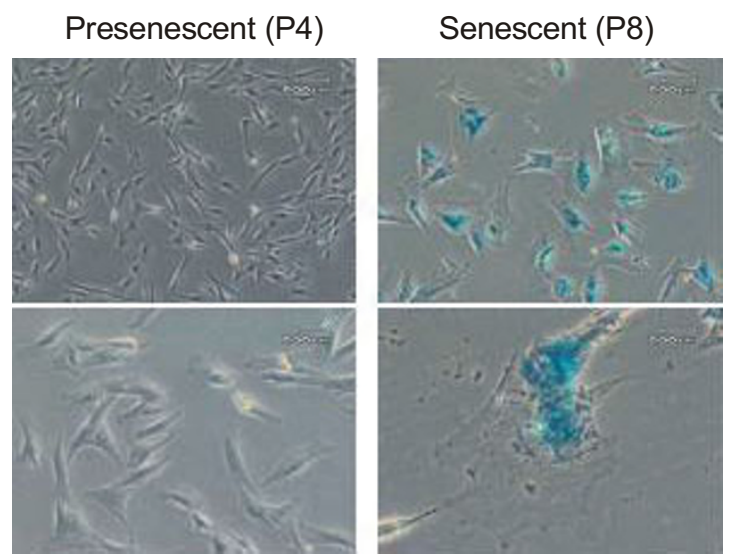

C

\section{D}
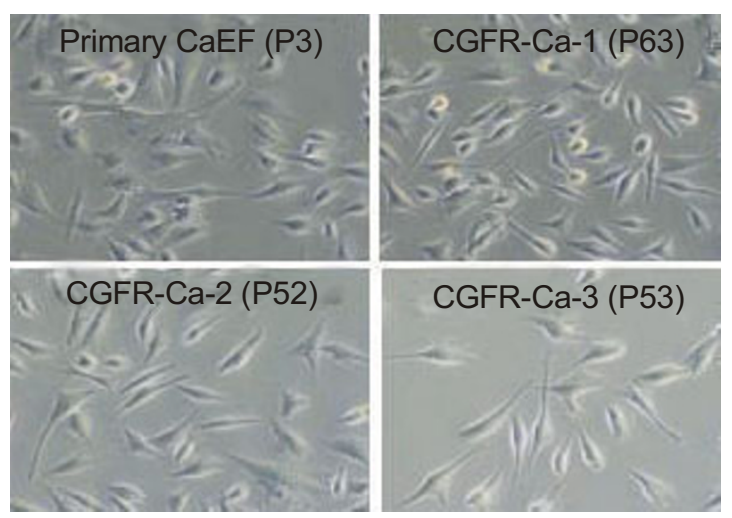

Figure 1. Growth kinetics and spontaneous immortalization of primary CaEF cells. (A) Cell population doubling levels (PDL) were determined in primary $\mathrm{CaEF}$ cells by $3 \mathrm{~T} 3$ cell culture protocol. Three arrows indicate presenescent, senescent and spontaneous immortalized passages of CaEF cells. (B) Representative photographs (upper photos, 40x; lower photos, 100x magnification) show primary presenescent passage 4 and senescent passage $8 \mathrm{CaEF}$ cells stained with $\mathrm{X}$-gal solution to determine senescence-associated $\beta$-galactosidase activity. $(\mathrm{C})$ Photograph (left) shows passage 10 senescent $\mathrm{CaEF}$ cells stained with Giemsa solution. Cells in each colony were eventually converted to immortal cells and the representative cells in one colony showing in the right photo (40x magnification). (D) Representative photographs (40x magnification) show the cell morphology of primary CaEF (passage 3), CGFR-Ca-1 (passage 63), CGFR-Ca-2 (passage 52) and CGFR-Ca-3 (passage 53) cells.

\section{Functional loss of p53 activity in immortal CaEF cell lines}

Since numerous studies have shown that p53 loss is usually necessary for lifespan extension, eventually leading to cellular immortal conversion (Sherr and DePinho, 2000), the expression of p53 mRNA and protein in each immortalized $\mathrm{CaEF}$ cell lines were determined. As shown in Figure 3A, the CGFR-Ca-1 and -3 cell lines showed barely detectable levels of p53 mRNA as compared to the CGFR-Ca-2 cell line. Consistent with the expression of p53 mRNA, p53 protein expression was barely detected in the CGFRCa-1 and -3 cell lines (Figure 3A). However, abundant expression of $p 53$ in the CGFR-Ca-2 cell line might be inactive as the p53 gene in this cell line was found with a single nucleotide alteration, leading to a single amino acid change in the DNA binding domain of p53 (data not shown). Cell viability was examined in order to determine the biological function of endogenous p53 in the primary $\mathrm{CaEF}$ and three immortal $\mathrm{CaEF}$ cell lines by treatment with different concentrations of adriamycin, a DNA-damage-inducing agent. As shown in Figure $3 \mathrm{~B}$, primary $\mathrm{CaEF}$ cells were shown to be quite sensitive to a low dosage of adriamycin treatment $(18 \%$ viable at $20 \mathrm{nM}$ adriamycin), while all three immortal $\mathrm{CaEF}$ cell lines displayed significant resistance to 20 $\mathrm{nM}$ adriamycin treatment $(57 \%$ viable in the CGFR$\mathrm{Ca}-1 ; 96 \%$ in the CGFR-Ca-2; $66 \%$ in the CGFRCa-3), suggesting that the three immortal CaEF cell lines might lose the p53 regulatory function. To further dissect whether p53 regulatory function loss is due to either to the loss of p53 itself or the loss of certain signal pathways regulated by $\mathrm{p} 53$ or both, the primary $\mathrm{CaEF}$ and three immortal $\mathrm{CaEF}$ cell lines were in- 

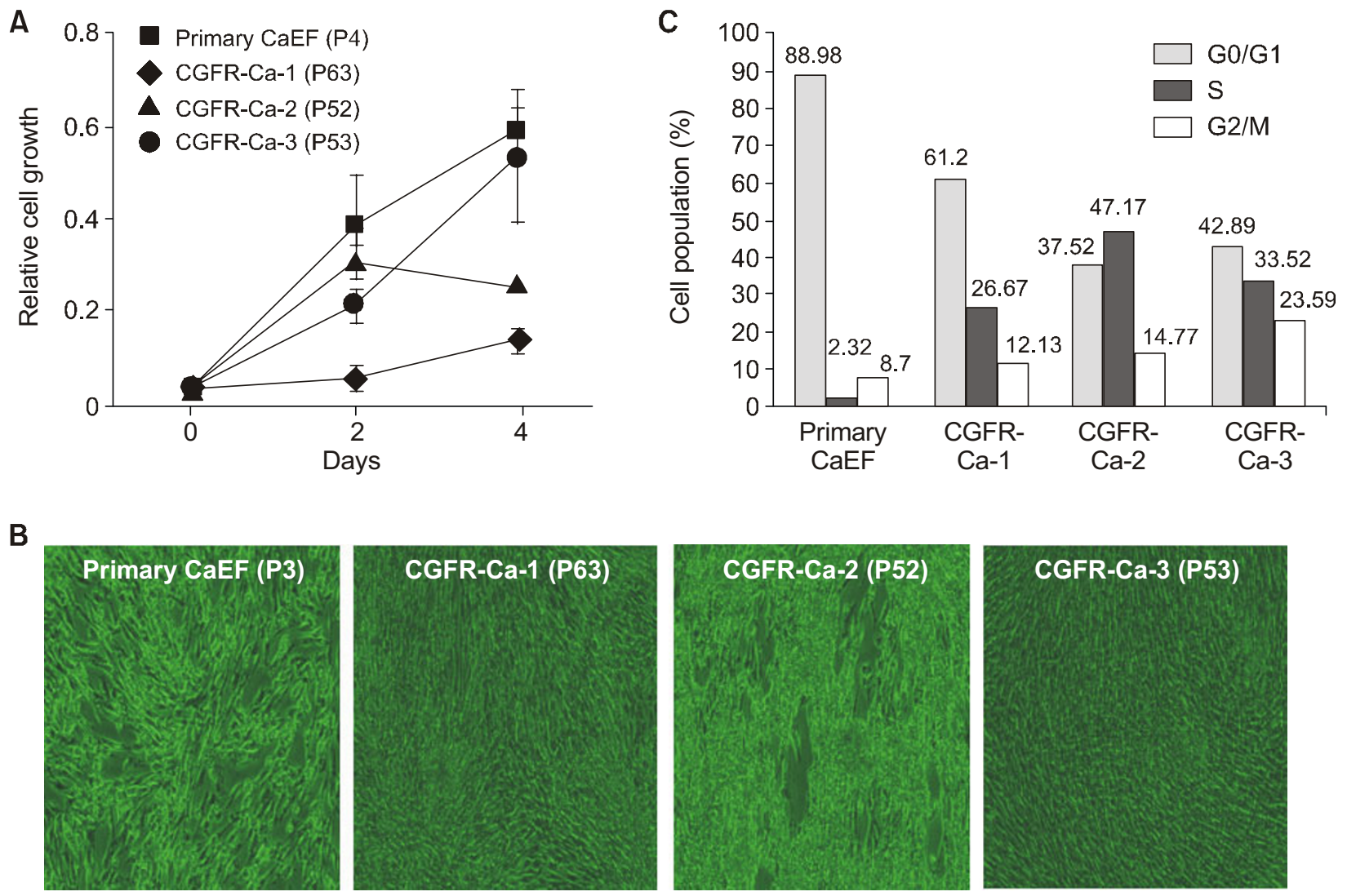

D

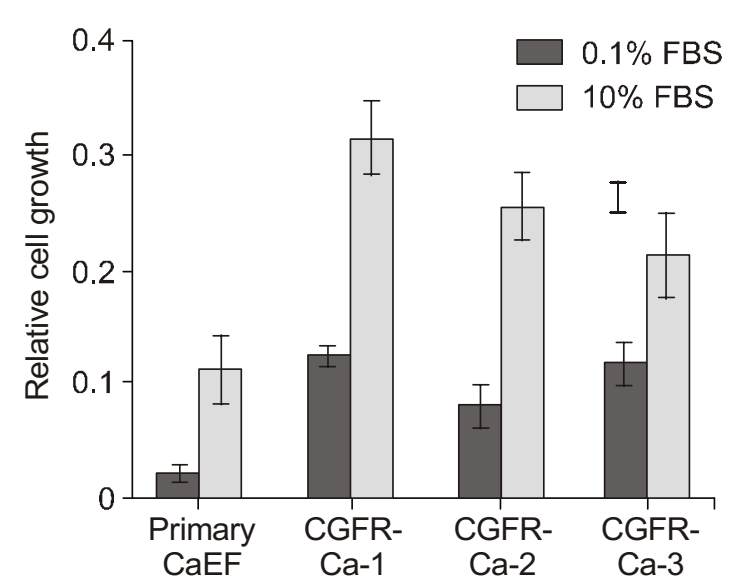

$\mathrm{E}$

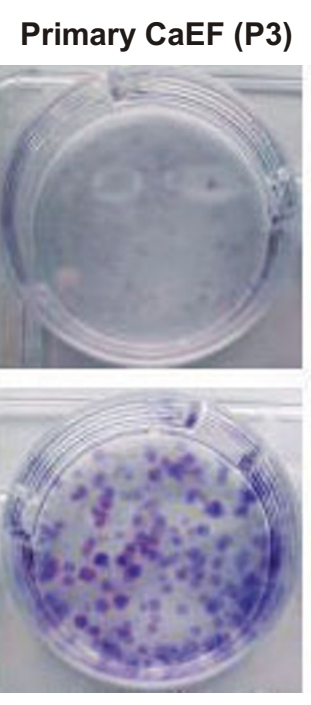

CGFR-Ca-2 (P52)

\section{CGFR-Ca-1 (P63)}
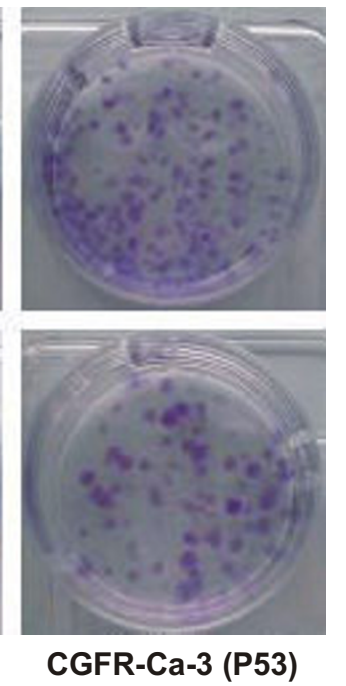

Figure 2. Growth characteristics of primary CaEF and immortal CaEF cells. (A) Primary CaEF (passage 4), CGFR-Ca-1 (passage 63), CGFR-Ca-2 (passage 52) and CGFR-Ca-3 (passage 53) cells grown in 10\% FBS-DMEM were stained with crystal violet solution every 2 days for 4 days to determine relative cell growth rates. (B) Representative photographs (40x) show cellular morphology of primary CaEF (passage 3), CGFR-Ca-1 (passage 63), CGFR-Ca-2 (passage 52) and CGFR-Ca-3 (passage 53) cells grown under confluent culture conditions. (C) For analysis of cell division rates, cell cycle profile of primary CaEF (passage 3), CGFR-Ca-1 (passage 63), CGFR-Ca-2 (passage 52) and CGFR-Ca-3 (passage 53) cells were performed using FACScan and Cell Quest software program. Mean percentages of cell population in G0/G1, S and G2/M phases of each cell are displayed above the corresponding bars. (D) Relative growth rates of primary $\mathrm{CaEF}$ and three immortal CaEF cells grown in $0.1 \%$ FBS-DMEM and 10\% FBS-DMEM for 3 days were determined by crystal violet staining. (E) Representative photographs show density-dependent growth rates of primary $\mathrm{CaEF}$ and three immortal $\mathrm{CaEF}$ cells plated with low-density cell population. These cells were grown in $10 \%$ FBS-DMEM for 14 days and stained with crystal violet solution. 
A
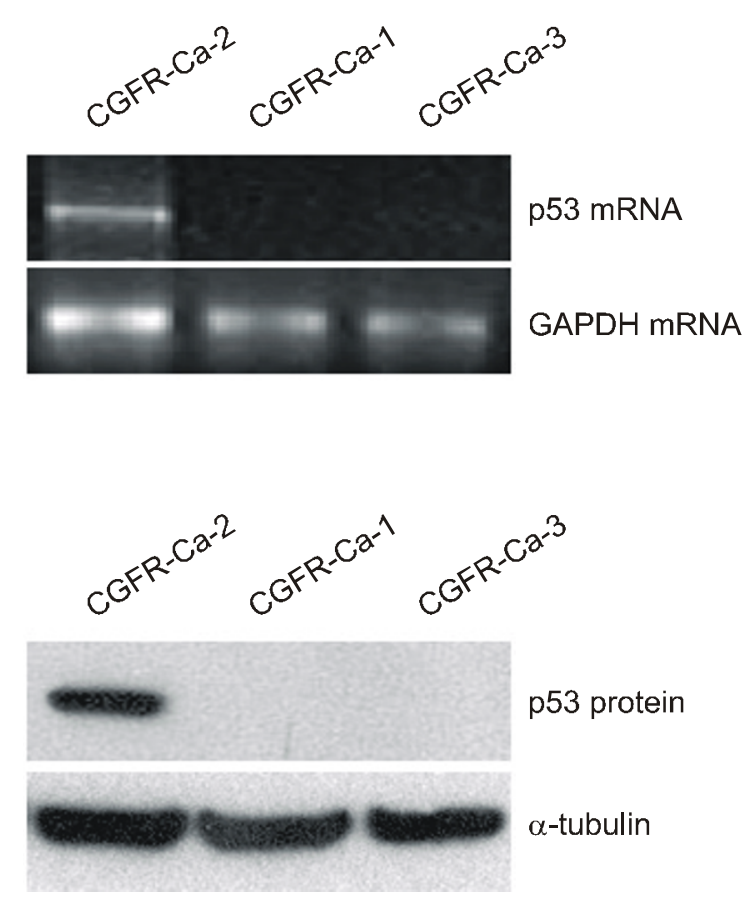

B

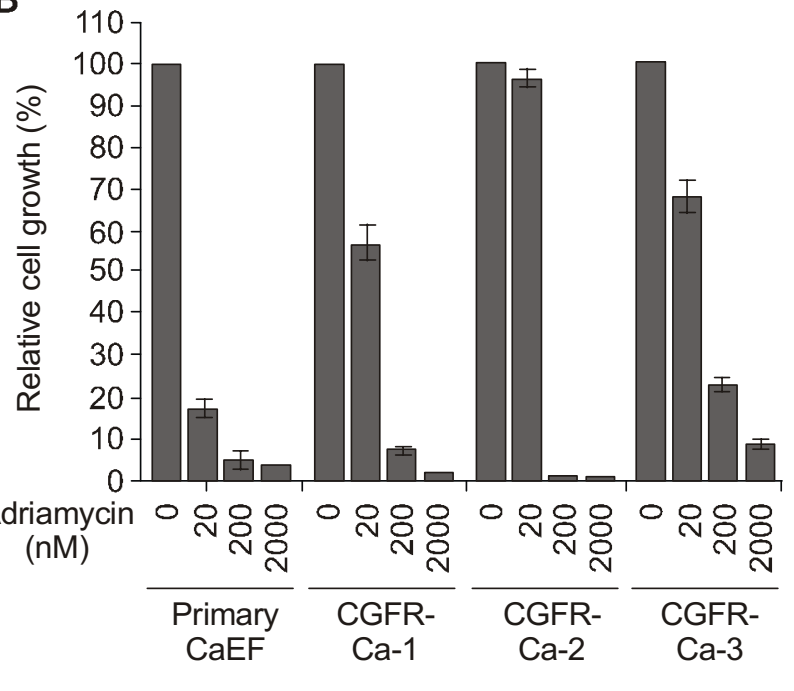

C

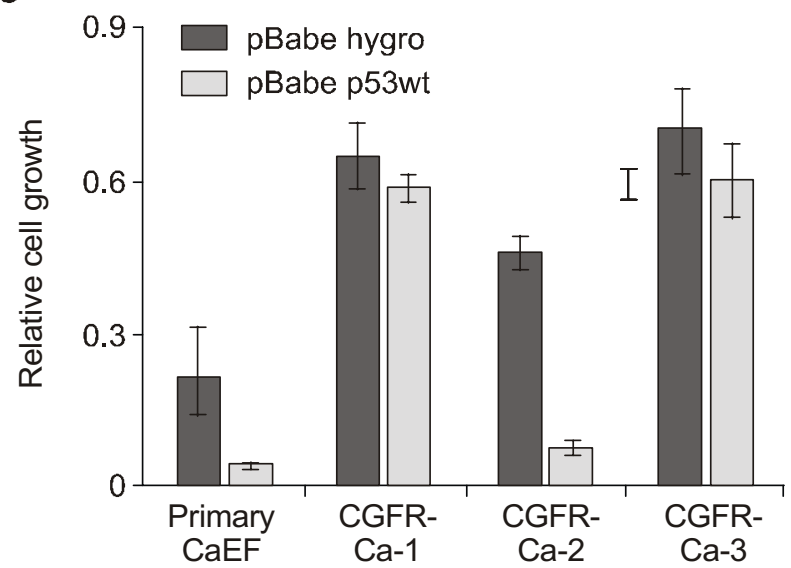

Figure 3. Functional loss of $p 53$ activity in immortal CaEF cells. (A) Expression of canine p53 mRNA and protein in the three immortal CaEF cell lines were determined by semi-quantitative RT-PCR and Western blot analysis, respectively. (B) Relative viability of primary CaEF and three immortal $\mathrm{CaEF}$ cells grown in the absence $(0 \mathrm{nM})$ or presence of adriamycin $(20,200$ and $2000 \mathrm{nM})$ for 3 days were determined by staining cells with crystal violet solution. (C) Relative growth rates of primary CaEF and three immortal CaEF cells infected with wild-type of p53 were determined by staining cells with crystal violet solution.

fected with retrovirus-expressing pBabe-hygromycin or pBabe-wild-type p53-hygromycin. As shown in Figure $3 \mathrm{C}$, primary $\mathrm{CaEF}$ and immortal CGFR-Ca-2 cell lines infected with wild-type p53 were found to grow slower than their counterparts infected with pBabe-hygromycin, while immortal CGFR-Ca-1 and -3 cell lines showed significant resistance to overexpression of wild-type p53.

\section{Loss of $\mathrm{p} 16^{\mathrm{INK} 4 \mathrm{a}}$ regulatory function in immortal CaEF cell lines}

Since ample evidence has shown that alteration in p16 INK4a/Rb pathways is a common genetic event occurring in cellular immortalization transformation
(Serrano and Blasco, 2001), the functionality of $\mathrm{p} 16^{\mathrm{INK} 4 \mathrm{a}} / \mathrm{Rb}$ pathways was determined in primary $\mathrm{CaEF}$ and the three immortal CaEF cell lines. $16^{\text {INK4a }}$ involved in hypophosphorylation of $\mathrm{Rb}$ and $\mathrm{G} 1$ checkpoint arrest is inactivated in many transformed cell lines. Transcriptional silencing of $p 16^{\text {INK4a }}$ in many immortal cells is due to the hypermethylation of its promoter (Merlo et al., 1995; Serrano, 1997). As shown in Figure 4A, in the CGFR-Ca-1 and -2 cell lines, expression of canine $\mathrm{p} 16^{\mathrm{INK} 4 \mathrm{a}} \mathrm{mRNA}$ was shown to be evidently detectable and was not even altered by treatment with DNA methylation inhibitor, 5-aza-2'deoxycytidine (5-aza-dC) (Kim et al., 2002b). However, p16 ${ }^{\text {INK4a }}$ mRNA was found to be dramatically decreased, while restored in immortal CGFR-Ca-3 
A
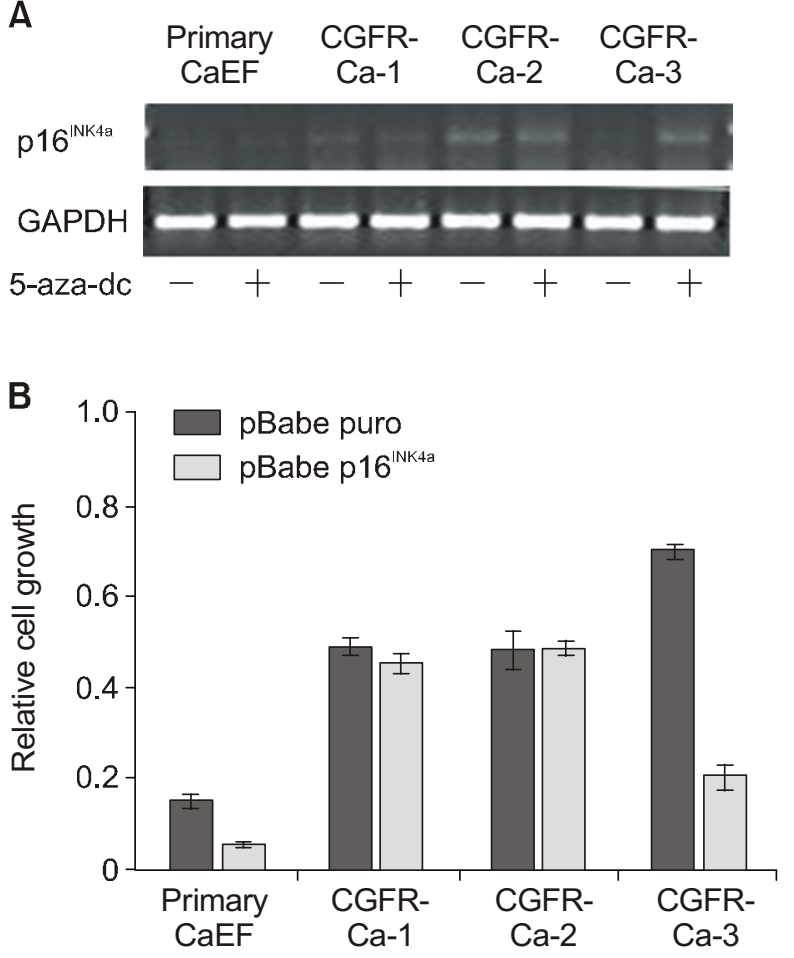

C
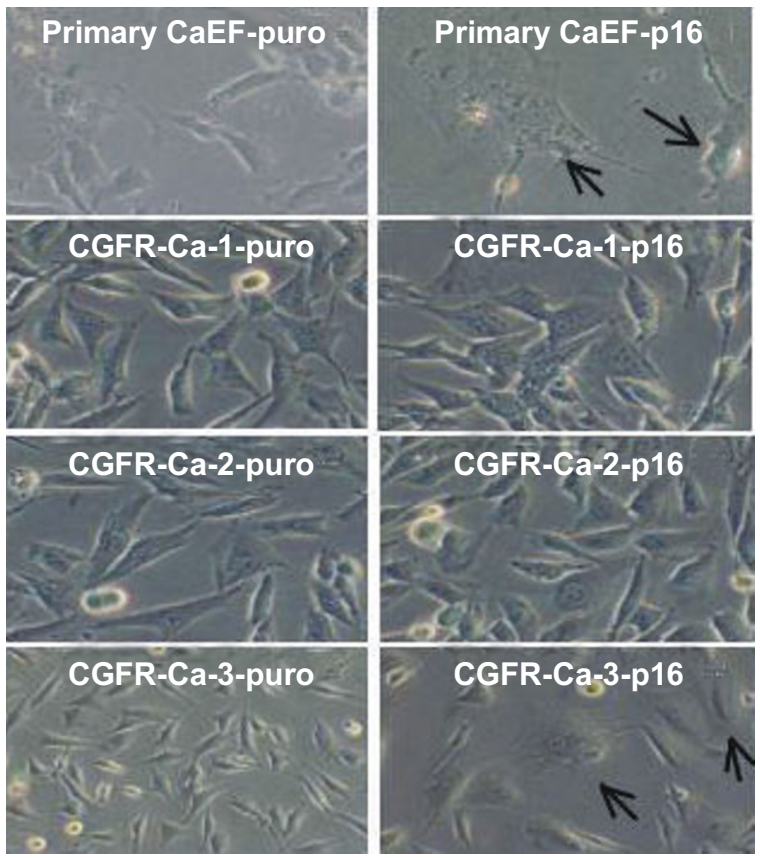

D

CGFR-Ca-1

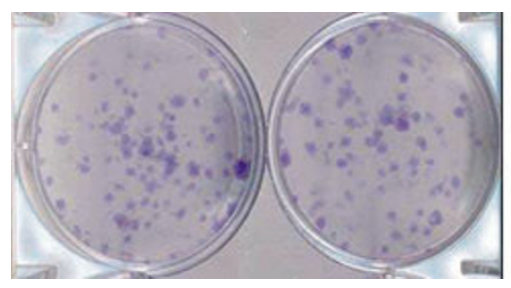

CGFR-Ca-2

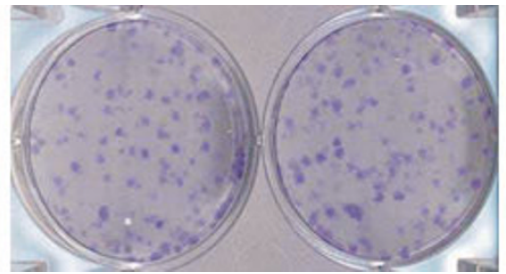

CGFR-Ca-3

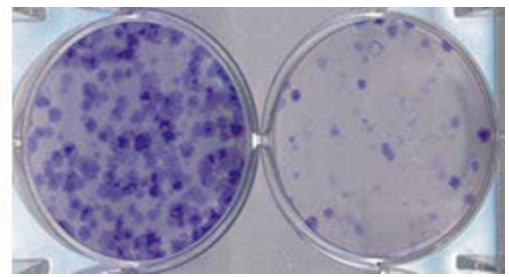

Figure 4. Functional loss of $p 16^{\text {inka }}$ regulation in immortal immortal CaEF cells. (A) Expression of endogenous canine $p 16^{\text {ink4a }}$ in primary CaEF (passage 4), CGFR-Ca-1 (passage 63), CGFR-Ca-2 (passage 52) and CGFR-Ca-3 (passage 53) cells treated with 5'-aza-2'-deoxycytidine was determined by semi-quantitative RT-PCR. (B) Relative growth rates of primary CaEF (passage 3), CGFR-Ca-1 (passage 63), CGFR-Ca-2 (passage 52) and CGFR-Ca-3 (passage 53) cells infected with wild-type of 553 were determined by staining cells with crystal violet solution. (C) Representative photographs (40x magnification) show cellular morphology of primary CaEF (passage 3), CGFR-Ca-1 (passage 63), CGFR-Ca-2 (passage 52) and CGFR-Ca-3 (passage 53) cells infected with pBabe-puromycin (left photos) and pBabe-p16 ${ }^{\text {INK4a }}$-puromycin vector (right photos). Arrows indicate cells representing typical senescent morphology. (D) Representative photographs show density-dependent growth rates of either pBabe-puromycin or pBabe-p $16^{\text {INK4a }}$-puromycin expressing three immortal CaEF cells plated with low-density cell population. These cells were grown in 10\% FBS-DMEM for 14 days and stained with crystal violet solution.

when treated with 5 -aza-dC, indicating that $\mathrm{p} 16^{\text {INK4a }}$ expression might be downregulated in CGFR-Ca-3 cells possibly due to hypermethylation of 'CpG island' in its promoter (Merlo et al., 1995). To further determine the biological significance of the downregulated $\mathrm{p} 16^{\mathrm{INK} 4 \mathrm{a}}$ in CGFR-Ca-3 cells, an exogenous human $\mathrm{p} 16^{\mathrm{INK} 4 \mathrm{a}}$ was transfected into primary $\mathrm{CaEF}$ and the three immortal $\mathrm{CaEF}$ cell lines using a retroviral transduction system. The result showed that cell growth in only the primary CaEF and CGFR-Ca-3 cells was significantly decreased by overexpression of human $\mathrm{p} 16^{\mathrm{INK} 4 \mathrm{a}}$, but not in CGFR-Ca-1 and -2 (Figure $4 \mathrm{~B})$. Both $\mathrm{p} 16^{\mathrm{INK} 4 \mathrm{a}}$ expressing primary $\mathrm{CaEF}$ and CGFR-Ca-3 cell morphology was quite similar to that shown in the typical senescent cell, such as multinuclear, enlarged and flattened structures (Figure 4C). In addition, cell colony formation under low-density seeding assay was dramatically decreased in only the CGFR-Ca-3 cells when $\mathrm{p} 16^{\mathrm{INK} 4 \mathrm{a}}$ was overexpressed, as compared to the CGFR-Ca- 1 and -2 cell lines 
A
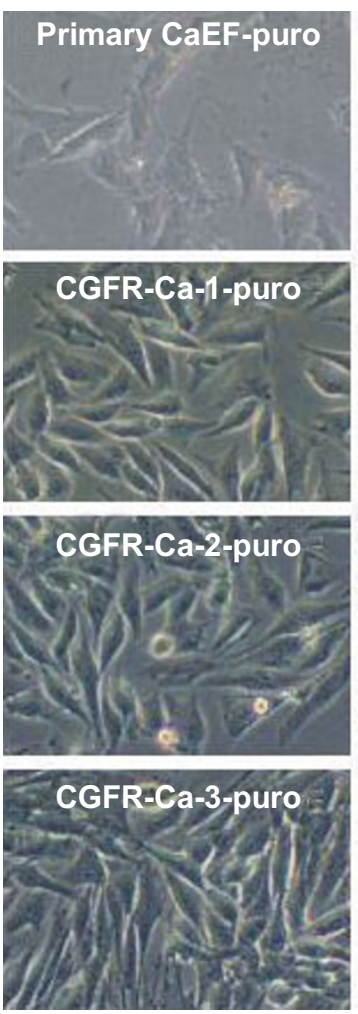
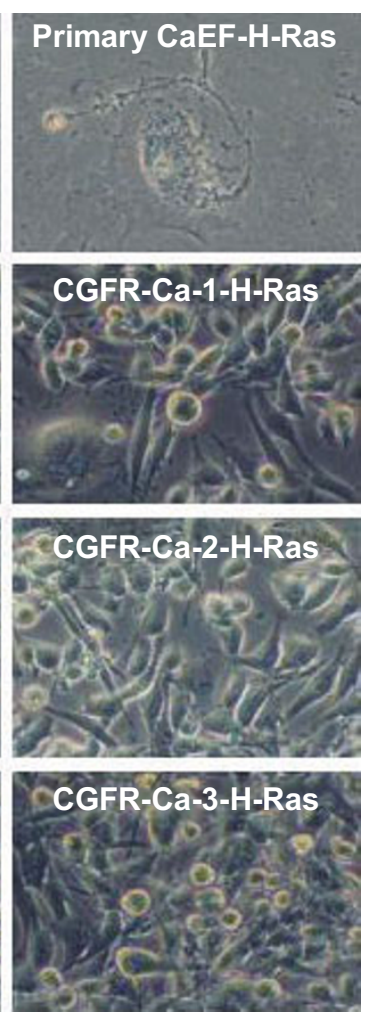

B
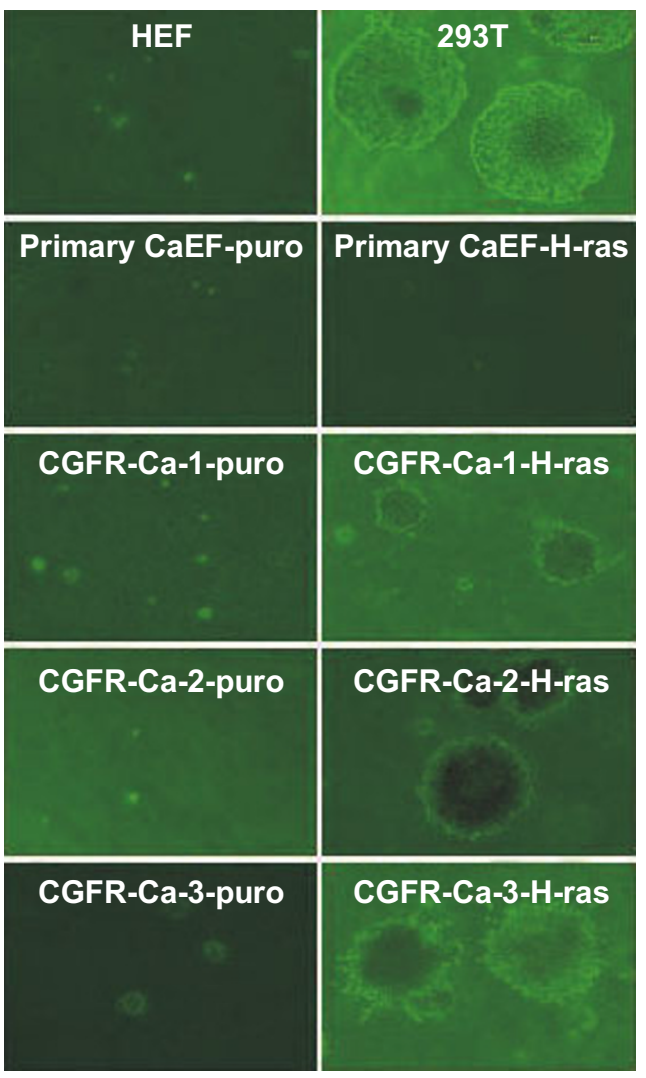

Figure 5. Oncogenic transformation of immortal CaEF cells by overexpression of oncogenic H-Ras ${ }^{\mathrm{V} 12}$. (A) Representative photographs (40x) show primary cells and three immortal CaEF cells stably infected with oncogenic H-Ras ${ }^{\mathrm{V} 12}$ (pBabe-H-Ras ${ }^{\mathrm{V} 12}$-puromycin) or control vector (pBabe-puromycin). (B) Representative photographs show cellular morphology of normal human embryonic fibroblast (HEF), transformed 293T, primary $\mathrm{CaEF}$ (infected with either pBabe-puromycin or pBabe-H-RasV12-puromycin) and three immortal $\mathrm{CaEF}$ cells (infected with either pBabe-puromycin or pBabe-H-RasV12-puromycin) grown in soft-agar dishes for 14 days.

(Figure 4D).

\section{Transformation of immortal CaEF cells by oncogenic H-Ras ${ }^{\mathrm{V} 12}$}

Overexpression of oncogenic $\mathrm{H}-\mathrm{Ras}^{\mathrm{V} 12}$ are known to induce premature senescence similar to replicative senescence in primary cells containing normal p53 function, and deficiency in p53 leads to a bypassing of H-Ras ${ }^{\mathrm{V} 12}$-induced senescence (Serrano et al., 1997). To determine the response of the immortal CGFR-Ca-1, -2 and -3 cell lines to oncogenic $\mathrm{H}$-Ras ${ }^{\mathrm{V} 12}$, primary $\mathrm{CaEF}$ and the three immortal CaEF cell lines were subjected to overexpression of oncogenic $\mathrm{H}-\mathrm{Ras}^{\mathrm{V} 12}$ using a retroviral transduction system. As shown in Figure 5A, primary $\mathrm{CaEF}$ cells overexpressing $\mathrm{H}-\mathrm{Ras}^{\mathrm{V} 12}$ became prematurely senescent as judged by cell morphology such as enlarged, flattened and highly vacuolated shapes. In contrast to primary $\mathrm{CaEF}$, the three immortal $\mathrm{CaEF}$ cell lines overexpressing oncogenic H-Ras ${ }^{\mathrm{V} 12}$ showed an in- creased mitotic cell population compared to cell lines overexpressing the control pBabe-puromycin vector, indicating that no evident senescence occurs in immortal CaEF cell lines overexpressing oncogenic $\mathrm{H}-\mathrm{Ras}^{\mathrm{V} 12}$. Furthermore, when the three immortal $\mathrm{CaEF}$ cell lines overexpressing oncogenic $\mathrm{H}-\mathrm{Ras}^{\mathrm{V} 12}$ were allowed to grow under soft-agar culture conditions, these cell lines converted to highly transformed cell colonies comparable to the aggressive transforming 293T cancer cell line (Figure 5B). However, normal human embryonic fibroblast (HEF), primary $\mathrm{CaEF}$ expressing either control pBabepuromycin or oncogenic $\mathrm{H}-\mathrm{Ras}^{\mathrm{V} 12}$ and the three immortal $\mathrm{CaEF}$ cell lines expressing control pBabepuromycin vector failed to convert to transforming cells under the soft-agar culture condition (Figure $5 \mathrm{~B}$ ), indicating that the three immortal CGFR-Ca-1, -2 and -3 cell lines do not possess the properties of transformed cells. 


\section{Discussion}

Similar to the primary murine embryonic fibroblast (MEF) cells, primary canine embryonic fibroblast (CaEF) cells showed a relatively short lifespan in vitro and frequent conversion to spontaneous immortal cells, when primary CaEF cells reached the end of their lifespan, replicative senescent stage. Spontaneous immortalization in human and chicken cells is extremely rare (Harris, 1987; Kim et al., 2001), whereas this event in mouse cells is known to occur by the order of magnitudes greater than the rate observed in human and chicken cells. Currently, there exist just a few reporting on the spontaneous immortalization of canine cells. We have established two spontaneously immortalized $\mathrm{CaEF}$ cell lines from five different canine embryos and one telomerase-mediating immortalized CaEF cell line. Our result demonstrates that spontaneous immortalization is a relatively frequent event in canine cells, as it is in mouse cells. It is well documented that cellular immortalization can be achieved by the loss of either p53 or a variety of p53-regulatory factors in human- and rodentderived cells (Sherr and DePinho, 2000). Upon various upstream-signals in response to DNA-damaging insults, p53 is able to activate and operate as a transcriptional activator to induce the expression of a variety of negative cell cycle regulators or proapoptotic regulators (Ko and Prives, 1996).

All three immortal $\mathrm{CaEF}$ cells used in the present study showed the inactivated p53 function. One CGFR-Ca-2 cell line was found to have a mutant p53 protein, while CGFR-Ca-1 and -3 cell lines did not express detectable levels of p53 mRNA and protein as determined by semi-quantitative RT-PCR and western blot analysis, respectively. Although the molecular mechanism leading to the downregulation of the p53 mRNA in the CGFR-Ca-1 and -3 cell lines is currently unclear, it is possible that p53 mRNA in the CGFR-Ca-1 and -3 cell lines might be rapidly degraded as shown in spontaneously immortalized avian cell lines observed in our previous data (Kim et al., 2001). The three immortal CaEF cell lines grown in the presence of adriamycin did not display evident DNA damage responses such as cell cycle arrest or cell death, while primary cell growth was significantly decreased by the same treatment. The finding that forced overexpression of wild-type p53 did not induce cell cycle arrest or cell death in immortal CGFR-Ca-1 and -3 cell lines could be explained by the CGFR-Ca-1 and -3 cell lines having unidentified mechanisms to rapidly degrade exogenous p53 as suggested by our previous study (Kim et al., 2001). However, like primary CaEF cells, immortal CGFRCa-2 cells exogenously transduced with wild-type p53 displayed a significant decrease in viable cell num- bers, indicating that the CGFR-Ca-2 cell line plausibly inactivated the p53 gene itself.

The overexpression of oncogenic H-Ras ${ }^{\mathrm{V} 12}$ is known to induce premature senescence similar to replicative senescence in primary human and mouse fibroblast cells containing normal p53 function (Serrano et al., 1997). Primary CaEF cells, like primary human and mouse fibroblast cells, are likely to possess a quite similar replicative senescent nature in response to oncogenic insults. Meanwhile, the deficiency in $\mathrm{p} 53$ leads to a bypassing of H-Ras ${ }^{\mathrm{V} 12}$ induced senescence and allows these cells to be transformed. As oncogenic $\mathrm{H}-\mathrm{Ras}^{\mathrm{V} 12}$ was overexpressed in the primary $\mathrm{CaEF}$ and three immortal CaEF cell lines, all three immortal $\mathrm{CaEF}$ cell lines were shown to be transformed, while the primary CaEF cells displayed prototypical replicative senescent morphology. These results suggest that our immortal CaEF cell lines by themselves should not be transformed but immortalized.

Immortalizing conversion in primary cells can occur through the loss of either $\mathrm{Rb}$ or its upstream- or downstream-regulatory factors (Sherr and DePinho, 2000). The ${ }^{\text {INK4a }}$ genetic locus encodes two structurally and functionally different proteins, $\mathrm{p} 16^{\mathrm{INK} 4 \mathrm{a}}$ and ARF (Kamijo et al., 1997). Whereas the ARF protein functions upstream of p53 by binding to MDM-2, preventing it from degrading p53 (Tao and Levine, 1999; Weber et al., 1999), p16 ${ }^{\text {INK4a }}$ acts upstream of Rb. Hyperphosphorylated $\mathrm{Rb}$ by D-type cyclins and cyclindependent kinase $4 / 6 \quad(\mathrm{CDK} 4 / 6)$ releases the transcription factor E2F-1, which is then free to activate genes necessary for the S-phase of the cell cycle (Dyson, 1998). The biological function of p16 ${ }^{\text {INK4a }}$ is known to inactivate CDK4/6, resulting in a hypophosphorylated $\mathrm{Rb}$ and $\mathrm{G} 1$ checkpoint arrest (Merlo et al., 1995; Sherr, 1996). Transcriptional silencing of $\mathrm{p} 16^{\mathrm{INK} 4 \mathrm{a}}$ in many immortal cells is due to hypermethylation of its promoter (Merlo et al., 1995). We did not determine the expression and biological function of canine p19ARF, which is currently not identified. However, the biological function of $\mathrm{p} 16^{\text {INK4a }}$ was evaluated for the $\mathrm{Rb}$ regulatory function in the three immortal $\mathrm{CaEF}$ cell lines. It appears that, unlike CGFR-Ca-3 that down-regulated p16 ${ }^{\text {INK4a }}$ mRNA due to its promoter methylation but had an intact $\mathrm{p} 16^{\text {INK4a }}$ regulatory pathway, CGFR-Ca-1 and -2 expressed p16 ${ }^{\text {INK4a }}$ tumor suppressor mRNA but had a functionally inactive $\mathrm{p} 16^{\mathrm{INK} 4 \mathrm{a}}$ regulatory pathway as determined by the lack of obvious differences in the cell growth and phenotype when reconstituted with wild-type $\mathrm{p} 16^{\mathrm{INK} 4 \mathrm{a}}$. These results suggest that CGFR-Ca-1 and -2 should have an inactive $\mathrm{p} 16^{\text {INK4a/ }}$ $\mathrm{Rb}$ signal pathway due to not $\mathrm{p} 16^{\text {INK4a }}$ itself but the p16 ${ }^{\text {INK4a }}$-downstream regulatory pathways.

Taken together, the three immortal CaEF cell lines 
shown in the present study appear to have a functional loss of $\mathrm{p} 53$ and $\mathrm{p} 16^{\text {INK4a }}$ in different regulatory pathways. This loss of $\mathrm{p} 53$ and $\mathrm{p} 16^{\mathrm{INK} 4 \mathrm{a}} / \mathrm{Rb}$ regulatory function might play a crucial role in cellular immortalization of canine embryonic fibroblast cells in much the same way as cells derived from other species.

\section{Acknowledgement}

This work was supported by a Korea University grant to S. You and a grant from Imgen Inc. (Seoul, South Korea) to H. Kim and S. You.

\section{References}

Attardi LD, Reczek EE, Cosmas C, Demicco EG, McCurrach $M E$, Lowe SW, Jacks T. PERP, an apoptosis-associated target of p53, is a novel member of the PMP-22/gas3 family. Genes Dev 2000;14:704-14

Aviv A, Harley CB. How long should telomeres be?, Curr Hypertens Rep 2001;3:145-51

Barak $Y$, Juven T, Haffner R, Oren M. Mdm2 expression is induced by wild type p53 activity. EMBO J 1993;12:461-8

Bodnar AG, Ouellette M, Frolkis M, Holt SE, Shiu CP, Morin GB, Harley CB, Shay JW, Lichtsteiner S, Wright WE. Extension of life-span by introduction of telomerase into normal human cells. Science 1998;279:349-52

Campisi J, Replicative senescence and immortalization, in: Stein G, Baserga R, Giordano A, Denhardt D (Eds). In The Molecular Basis of Cell Cycle and Growth Control, Wiley-Liss, New York, 1999,348-73

Dimri GP, Lee X, Basile G, Acosta M, Scott G, Roskelley C, Medrano EE, Linskens M, Rubelj I, Pereira-Smith O, Peacocke M, Campisi J. A biomarker that identifies senescent human cells in culture and in aging skin in vivo. Proc Natl Acad Sci USA 1995;92:9363-7

Dyson N. The regulation of E2F by pRB-family protein. Genes Dev 1998;12:2245-62

El-Diery W, Tokino T, Velculescu V, Levy D, Parsons R, Trent J, Lin D, Mercer E, Kinzler K, Vogelstein B. WAF1, a potential mediator of p53 tumor suppression. Cell 1993; 75:817-25

Felsher DW, Bishop JM. Transient excess of MYC activity can elicit genomic instability and tumorigenesis. Proc Natl Acad Sci USA 1999;96:3940-4

Greider CW. Telomere length regulation. Annu Rev Biochem 1996;65:337-65

Harris CC. Human tissues and cells in carcinogenesis research. Cancer Res 1987;47:1-10

Hayflick L, Moorehead PS. The serial cultivation of human diploid cell strains. Exp Cell Res 1961;25:585-621

Kamijo T, Zindy F, Roussel MF, Quelle DE, Downing JR, Ashmun RA, Grosveld G, Sherr CJ. Tumor suppression at the mouse ${ }^{\text {INK4a }}$ locus mediated by the alternative reading frame product p19 ${ }^{A R F}$. Cell 1997;91:649-59

Kim H, You S, Kim I-J, Foster LK, Farris J, Ambady S, Ponce de Leon FA, Foster DN. Alterations in p53 and E2F-1 function common to immortalized chicken embryo fibroblasts. Oncogene 2001;20:2671-82

Kim H, You S, Farris J, Kong BW, Christman SA, Foster LK, Foster DN. Expression profiles of p53, p16 ${ }^{\text {INK4a }}$ and telomere regulating genes in the replicative senescent human, mouse and chicken fibroblast cells. Exp Cell Res 2002a;272:199-208

Kim H, Farris J, Christman SA, Kong BW, Foster LK, O'Grady SM, Foster DN. Events in the immortalizing process of primary human mammary epithelial cells by the catalytic subunit of human telomerase. Biochem J 2002b;365:765-72

Ko LJ, Prives C. p53: puzzle and paradigm. Genes Dev 1996;10:1054-72

Merlo A, Herman JG, Mao L, Lee DJ, Gabrielson E, Burger $P C$, Baylin SB, Sidransky D. 5' CpG island methylation is associated with transcriptional silencing of the tumor suppressor $\mathrm{p} 16 / \mathrm{CDKN} 2 / \mathrm{MTS} 1$ in human cancers. Nat Med 1995;88:686-92

Miyashita T, Reed JC. Tumor suppressor p53 is a direct transcriptional activator of the human bax gene. Cell 1995; 80:293-9

Oda $\mathrm{E}$, Ohki $\mathrm{R}$, Murasawa $\mathrm{H}$, Nemoto $J$, Shibue $T$, Yamashita T, Tokino T, Taniguchi T, Tanaka N. Noxa, a $\mathrm{BH} 3-$ only member of the $\mathrm{Bcl}-2$ family and candidate mediator of p53-induced apoptosis. Science 2000;288:1053-8

Sedivy JM. Can ends justify the means?: Telomeres and the mechanisms of replicative senescence and immortalization in mammalian cells. Proc Natl Acad Sci USA 1998;95:9078-81

Serrano M, Hannon G, Beach D. A new regulatory motif in cell-cycle control causing specific inhibition of cyclin D/CDK4. Nature 1993;366:704-7

Serrano M. The tumor suppressor protein $\mathrm{p} 16^{\text {INK4a }}$. Exp Cell Res 1997;237:7-13

Serrano M, Lin AW, McCurrach ME, Beach D, Lowe SW. Oncogenic ras provokes premature cell senescence associated with accumulation of p53 and p16 ${ }^{\text {INK4a }}$. Cell 1997;88: 593-602

Serrano M, Blasco MA. Putting the stress on senescence. Curr Opin Cell Biol 2001;13:748-53

Sherr CJ. Cancer Cell Cycles. Science 1996;274:1672-7

Sherr CJ, DePinho RA. Cellular senescence: mitotic clock or culture shock?. Cell 2000;102:407-10

Tao W, Levine AJ. ARF stabilizes p53 by blocking nucleocytoplasmic shuttling of Mdm2. Proc Natl Acad Sci USA 1999;96:6937-41

You S, Heo M, Moon J-H, Kim S-C, Kwak S, Yoon D-H, Jin D, Hong K-C, Foster DN, Choi Y-J, Kim H. Establishment of life-span extended bovine fibroblast cells carrying the characterization of primary cells. Mol Cells 2004; in press Weber J, Taylor L, Roussel M, Sherr CJ, Bar-Sagi D. 
Nucleolar Arf sequesters Mdm2 and activates p53. Nat Cell Biol 1999;1:20-6

Woods DB, Vousden KH. Regulation of p53 function. Exp
Cell Res 2001;264:56-66

Wu XW, Bayle JH, Olson D, Levine AJ. The p53 mdm-2 autoregulatory feedback loop. Genes Dev 1993;7:1126-32 